\title{
How often are angiotensin II and aldosterone concentrations raised during chronic ACE inhibitor treatment in cardiac failure?
}

\author{
R J MacFadyen, A F C Lee, J J Morton, S D Pringle, A D Struthers
}

\begin{abstract}
Objective-Angiotensin II (AII) and aldosterone are not always fully suppressed during chronic angiotensin converting enzyme (ACE) inhibitor treatment. In congestive heart failure (CHF) such failure of hormonal suppression is associated with increased mortality. This study examined how common AII and aldosterone increases are observed during routine clinical practice.

Patients and methods-91 patients with symptomatic (mean New York Heart Association class 2.7) CHF (mean (SD) left ventricular ejection fraction 29.9 (8)\%, range 9-46\%) were studied 4-6 hours after ACE inhibitor dosing. A representative range of ACE inhibitors (enalapril, lisinopril, captopril, perindopril, and fosinopril) was examined.
\end{abstract}

Results-Supine measurements showed a wide range of AII (10.5 (25.5) $\mathrm{pg} / \mathrm{ml})$, aldosterone (130.8 (136) $\mathrm{pg} / \mathrm{ml})$, and serum ACE (12.1 (13.3) EU/1; excludes captopril data) concentrations on diuretics. AII concentrations $>10 \mathrm{pg} / \mathrm{ml}$ were seen in $15 \%$ of patients, and aldosterone concentrations $>144 \mathrm{pg} / \mathrm{ml}$ were seen in $38 \%$ of patients. AII concentrations were significantly correlated $(p<0.001)$ with ACE but not with aldosterone concentrations. Aldosterone concentrations were not significantly correlated with ACE concentrations.

Conclusions-AII "reactivation" occurred in $15 \%$ and failure of aldosterone suppression in $38 \%$ of routine $\mathrm{CHF}$ patients taking ACE inhibitor treatment. AII "reactivation" was associated with both low and high levels of ACE activity, which suggests that multiple different mechanisms are at play. In patients with high plasma ACE concentrations, noncompliance should be considered along with inadequate dose titration. In patients with low plasma ACE and high AII concentrations, non-ACE mediated production of AII may be operative. Raised aldosterone concentrations appear to be more common than AII "reactivation". It is important to establish the cause of detectable or increased AII concentrations in a heart failure patient treated with an ACE inhibitor. The measurement of serum ACE may help to identify the likely cause as poor compliance or inadequate dose.

(Heart 1999;82:57-61)
Keywords: heart failure; hormone suppression; angiotensin II; aldosterone; angiotensin converting enzyme inhibitors; compliance

The effectiveness of angiotensin converting enzyme (ACE) inhibitors in reducing heart failure mortality ${ }^{1}$ may be largely attributable to hormone suppression. ${ }^{23}$ However, the reductions in angiotensin II (AII) and aldosterone may not be maintained with chronic ACE inhibitor treatment. While acute treatment with an ACE inhibitor can virtually eliminate AII from the plasma, ${ }^{4}$ chronic treatment has been associated with the reappearance of measurable AII in some patients. ${ }^{5}$ This has been called AII "reactivation" and is associated with a poor prognosis. ${ }^{67}$ It is also known that aldosterone does not remain suppressed during chronic ACE inhibitor treatment and this phenomenon has been called aldosterone escape.

While AII "reactivation" and aldosterone escape have been identified in the setting of clinical trials, very little is known about their incidence in routine clinical practice. The main aim of this study was to examine the prevalence of AII and aldosterone escape in an unselected group of patients with congestive heart failure (CHF) treated with ACE inhibition where compliance and dose titration are less rigorous than in clinical trials. We assessed the degree of neurohormonal suppression of aldosterone and AII in unselected patients with stable heart failure during routine treatment and examined the relation between them in order to examine possible mechanisms of AII "reactivation".

\section{Patients and methods}

Patients were recruited from an outpatient heart failure clinic or during hospitalisation for coincidental illness $(n=25)$ unrelated to chronic cardiac failure (for example, minor surgery, investigational or diagnostic procedures, intermittent reversible myocardial ischaemia). All patients were being treated for chronic heart failure with stable doses of diuretic and ACE inhibitor. Diagnosis had been made on the basis of medical history, ongoing symptoms, and physical examination and had been confirmed by a one or other of radionuclide scintigraphy $(n=63 ;$ MUGA ejection fraction $34(8) \%$, range $9 \%-46 \%$ ), contrast ventriculography ( $n=22$; qualitative comments made on the ventriculogram report), or echocardiography $(n=75$; mean fractional shortening 22 (5)\%, range $8-35 \%$ ). Some patients had multiple investigations. Details of patient demography, aetiology of 
Table 1 Patient demography and baseline treatment

\begin{tabular}{lcc}
\hline & Mean or $n$ & $S D$ \\
\hline Total number & 91 & \\
Male & 78 & \\
Body mass index $\left(\mathrm{kg} / \mathrm{m}^{2}\right)$ & 32 & 6 \\
Age (years) & 69 & 9 \\
Cause of CHF & & \\
$\quad$ Previous infarction & 84 & \\
Idiopathic dilated cardiomyopathy & 12 & \\
AF and hypertensive heart disease & 5 & \\
Left ventricular ejection fraction (\%) & 29.8 & 9 \\
Treatment & & \\
Frusemide (mg/day) & 67 & 57 \\
ACE inhibitor & 43 & \\
$\quad$ Enalapril & 35 & \\
$\quad$ Lisinopril & 10 & \\
$\quad$ Captopril & 2 & \\
$\quad$ Perindopril & 1 & \\
$\quad$ Fosinopril & 49 & 13 \\
Nitrate (n = 35) (mg/day) & 0.155 & 0.06 \\
Digoxin (n = 31) (mg/day) & 132 & 67 \\
Aspirin (n=39) (mg/day) & & \\
\hline AF, atrial fibrillation. & &
\end{tabular}

heart failure, and cardiac treatments are listed in table 1 .

DRUG TREATMENT

All subjects had received a constant dosage of ACE inhibitor for not less than four weeks before study and had had no changes to diuretic regimen. A variety of ACE inhibitors was being used. ACE inhibitor treatment was normalised to an equivalent dose in $\mathrm{mg}$ of enalapril per day using accepted pharmacological bioequivalence relations. ${ }^{8}$

PROCEDURE

The protocol for the study was passed by the local research and ethics committee before recruitment. All patients received an information leaflet before giving written and informed consent to participate in the study.

Patients were studied between 11:00 and 13:00 after routine self administration of drug treatment (approximately 4-6 hours after ACE inhibitor dosing). Intentionally no inquiries were made as to compliance with any treatment.

On reporting for the study, patients were weighed, underwent symptomatic inquiry and physical examination, and had a resting ECG documented. Patients were then rested supine (minimum 30 minutes) after insertion of a heparinised venous cannula in an antecubital vein to facilitate blood sampling.

Blood samples $(40 \mathrm{ml})$ for hormone and electrolyte studies were drawn rapidly into chilled syringes and distributed into appropriate aliquots with inhibitors (see below) on ice. Samples were immediately centrifuged at $2-3^{\circ} \mathrm{C}$ at approximately $3500 \mathrm{rpm}$, and serum or plasma separated and stored at $-70^{\circ} \mathrm{C}$ for later batched hormone analyses (within six months). Samples for serum electrolytes were processed on the day of collection.

LABORATORY METHODS OF ANALYSIS

Serum ACE activity

ACE was determined using direct radioimmunoassay ${ }^{9}$ operating within $2 \%$ intraand $4 \%$ interassay coefficient of variation and with a lower limit of detection of $0.5 \mathrm{EU} / 1$.
Table 2 Total sample measured hormones (mean (SD))

\begin{tabular}{llll}
\hline & $\begin{array}{l}\text { ACE activity } \\
(E U / l)^{\star}\end{array}$ & $\begin{array}{l}\text { Aldosterone } \\
(\mathrm{pg} / \mathrm{ml})\end{array}$ & AII $(\mathrm{pg} / \mathrm{ml})$ \\
\hline On diuretic & $12.1(13.3)$ & $130.8(136)$ & $10.5(25.5)$ \\
\hline *Excluding patients receiving oral captopril.
\end{tabular}

Plasma angiotensin peptides

Samples were collected into a chilled inhibitor cocktail containing renin inhibitor (enalkiren), ACE inhibitor (enalaprilat), and phenyl methy sulpho ethionamide before the separation of plasma to prevent further in vitro generation or interconversion of angiotensins. Preparative column chromatography was conducted according to the methodology of Nussberger and colleagues ${ }^{10}$ before the determination of angiotensin I (AI) and AII by conventional radioimmunoassay (Cardiovascular Assays, Glasgow, UK) using antiserum with minimal AI/AII cross reactivity. ${ }^{11}$ Inter- and intra-assay coefficients of variation were $4.5 \%$ and $9 \%$, respectively, for both $\mathrm{AI}$ and AII.

\section{Serum and urinary electrolytes}

Samples and aliquots were assayed on the day of collection using standard autoanalyser technology in our routine hospital clinical chemistry service operating with quality control standards $( \pm 2.5 \%$ interassay coefficient of variation).

Plasma aldosterone

Samples of plasma were analysed from frozen using a commercial radioimmunoassay (Peninsula Laboratories, California, USA).

STATISTICAL METHODS

Statistical analyses were conducted using the SPSS program. ${ }^{12}$ Linear correlation (Pearson) was assessed, and analysis of variance (ANOVA) and covariance (ANCOVA) with Bonferroni correction were applied to investigate differences between samples where relevant. All data are given as mean (SD).

\section{Results}

SERUM HORMONES

In the whole sample there was a large variation in measured hormone values for each of the parameters studied (table 2).

\section{Serum ACE}

Patients taking captopril $(\mathrm{n}=10)$ have been excluded from analyses involving ACE. Captopril's poor affinity for the enzyme in vitro makes serum ACE concentrations an inaccurate representation of the in vivo situation. ${ }^{13}$ During treatment with ACE inhibition in the doses outlined there was an appreciable failure of suppression of serum ACE activity during the period at which peak inhibitory effect would be anticipated - that is, 4-6 hours after dosing (fig 1). The distribution of measured serum ACE activity was wide. During chronic diuretic treatment one third of patients (27 of 81) had undetectable ACE (<2 EU/1), one third (26 of 81) measurable ACE activity (2-10 EU/1), and one third (28 of 81$)$ unsuppressed (> $10 \mathrm{EU} / \mathrm{l}) \mathrm{ACE}$. 


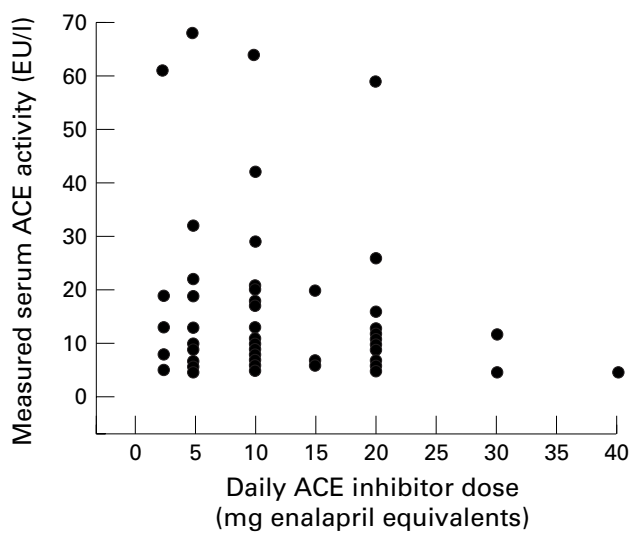

Figure 1 Relation between measured ACE activity and normalised ACE inhibitor dose (excluding those patients receiving oral captopril).

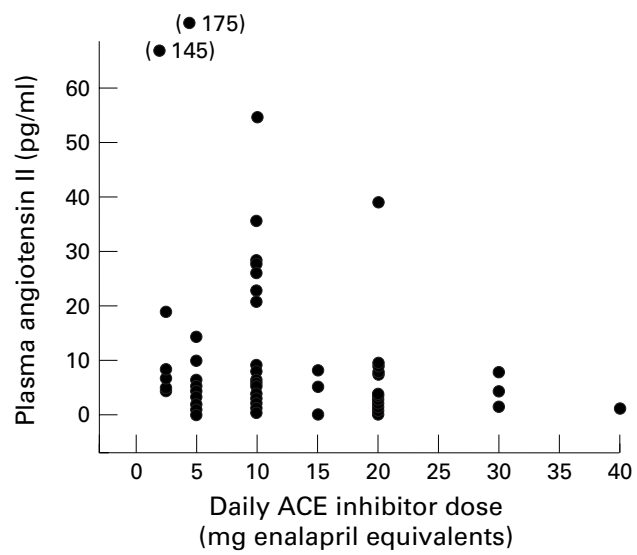

Figure 2 Relation between measured AII and normalised ACE inhibitor dose.

\section{Serum AII}

Considering the whole sample including captopril treated patients, there was again a notable variation in measured concentrations (fig 2). During chronic treatment full suppression of AII to very low concentrations $(<2 \mathrm{pg} / \mathrm{ml})$ was seen in nearly one third of patients (28 of 91 ). In these patients it may be said that AII had been eliminated from serum by treatment. There was some suppression below normal $(1-10 \mathrm{pg} / \mathrm{ml})$ in 45 of 91 (49\%) patients. Normal or modestly raised AII $(10-20 \mathrm{pg} / \mathrm{ml})$ concentrations were seen in 3 of 91 (3\%) patients, and increased AII ( $>20 \mathrm{pg} / \mathrm{ml}$ ) concentrations were seen in 10 of $91(11 \%)$ of the sample. Again these values are seen despite chronic drug treatment.

\section{Serum aldosterone}

Including the patients receiving captopril, supine aldosterone showed a wide spectrum of measured values (fig 3). Some patients had considerable increases of aldosterone regardless of ACE inhibitor or diuretic treatment. In one patient the measured concentration (on diuretic treatment) was in the range associated with primary aldosteronism (> $1000 \mathrm{pg} / \mathrm{ml}$ ). During chronic treatment suppression of aldosterone $(<144 \mathrm{pg} / \mathrm{ml})$ was noted in 56 of 91 patients $(61 \%), 35$ of $91(38 \%)$ had raised

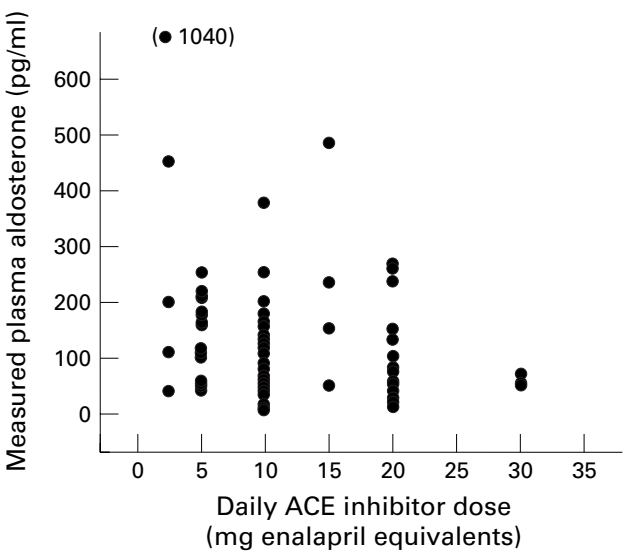

Figure 3 Relation between measured aldosterone and normalised ACE inhibitor dose.

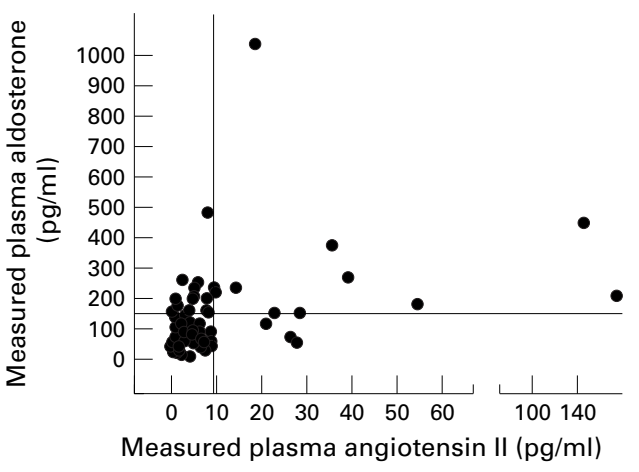

Figure 4 Relation between measured aldosterone and measured AII in same sample.

aldosterone (> $144 \mathrm{pg} / \mathrm{ml})$, and 13 of $91(14 \%)$ had notably increased aldosterone $(>300 \mathrm{pg} /$ $\mathrm{ml}$ ).

RELATION BETWEEN ACE ACTIVITY, AII, ALDOSTERONE, AND ACE INHIBITOR TREATMENT Looking at the associations between the measured parameters, plasma ACE activities were very variable at any given dose of $\mathrm{ACE}$ inhibitor (fig 1). The same was true for both aldosterone and AII (figs 2 and 3). Low doses of ACE inhibitor (equivalent of $2.5-5 \mathrm{mg}$ enalapril/day) was seen to produce excellent AII and aldosterone suppression in some individuals.

There was no significant relation between measured AII and aldosterone (fig 4). If AII "reactivation" is assumed to be a measured value of $>10 \mathrm{pg} / \mathrm{ml}$, despite chronic ACE inhibitor treatment, it occurred in the presence of a wide range of measured ACE activities. In those patients with AII concentrations $>10 \mathrm{pg} /$ $\mathrm{ml}$ approximately $50 \%$ had a plasma ACE activity $>20 \mathrm{EU} / 1$.

There was a significant correlation $(\mathrm{p}<0.001)$ between plasma AII and plasma ACE concentrations (fig 5). There was no significant correlation between plasma aldosterone and ACE concentrations (fig 6). Figures 5 and 6 also show that AII "reactivation" and aldosterone escape can both occur at a wide range of different, prevailing concentrations of ACE. 


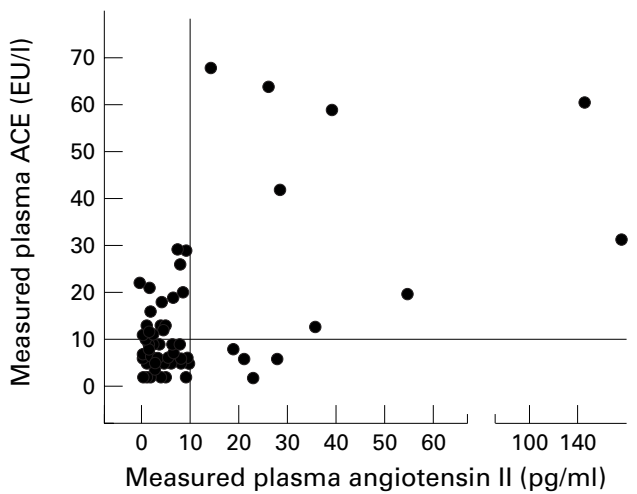

Figure 5 Relation between measured AII and ACE activity. There was a significant $(\mathrm{r}=0.75, p<0.001)$ correlation.

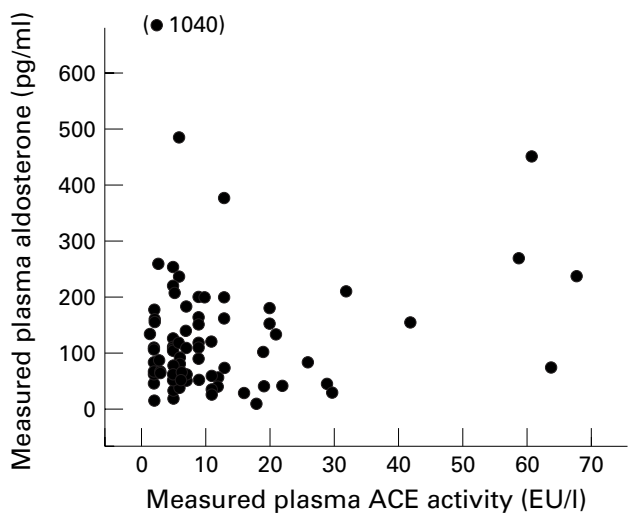

Figure 6 Relation between measured aldosterone and ACE activity. No significant correlation found.

\section{Discussion}

Large trial databases confirm that poor AII and aldosterone suppression are associated with an adverse outcome in individual heart failure patients. ${ }^{13}$ The only current data on the frequency of hormone "reactivation" come from subsets of these trials. As clinical trials only include highly selected patients all usually taking one drug in one dose, our observations in routine clinical practice are both novel and important.

The quantitative limits of hormone "reactivation" have not been defined. We selected AII concentration $>10 \mathrm{pg} / \mathrm{ml}$ and found this in approximately $15 \%$ of unselected patients. Raised aldosterone was defined as $>144 \mathrm{pg} /$ $\mathrm{ml}$. During chronic ACE inhibitor and diuretic treatment this occurred in a higher proportion of patients $(38 \%)$. This may simply relate to the fact that mechanisms other than AII are known to influence aldosterone concentrations. Indeed a very recent clinical trial in which spironolactone was added to an ACE inhibitor in the treatment of heart failure has been halted prematurely because of a mortality reduction advantage with spironolactone. ${ }^{14}$ Our results showing that aldosterone escape is more common than AII "reactivation" is consistent with this new clinical trial result.

We found that raised AII concentrations occurred with both low and high levels of serum ACE activity. This suggests that different mechanisms may be associated with AII "reactivation" in different patients. Serum
ACE activity is a very sensitive marker of the presence of an ACE inhibitor drug. Patients with high ACE activity (>10 EU/l) and AII "reactivation" may have this pattern because of poor compliance or inadequate individual dose titration of the ACE inhibitor. In patients with raised AII concentrations and low or undetectable ACE, non-ACE mediated AII generation or "reactivation" may be responsible.

Our findings have direct relevance to therapeutic strategies designed to maximise AII suppression. In patients with AII "reactivation" with high (>20 EU/l) or detectable (10$20 \mathrm{EU} / 1)$ ACE concentrations, it may be appropriate to either increase the dose of ACE inhibitor or to check compliance by assessing the biochemical response (ACE activity 4-5 hours after dosing) to supervised administration of the current ACE inhibitor dose. Clearly if poor compliance was identified (ACE eliminated below detection limit on single supervised dose) then the correct response would be to enhance compliance rather than increase the ACE inhibitor dose or change to an alternative agent, such as an AII antagonist. The only practical exception to this would be if the noncompliance was specifically related to ACE inhibitor cough. For those with AII "reactivation" but low or undetectable ACE it may be appropriate to change from an ACE inhibitor to an $\mathrm{AT}_{1}$ receptor antagonist or consider the combination of these treatments. These patients may be generating AII by non-ACE mediated catalysis of the breakdown of AI to AII. While this alternative AII generating system has been well demonstrated at a biochemical and cellular level, its importance in integrated human pathology or physiology is as yet unclear.

The cut off values we selected for both AII and aldosterone are inevitably arbitrary. The reason for this is simply that no quantitative definition of hormone "suppression" exists despite much clinical evidence of the overall importance of failure to suppress either hormone.

Serum ACE activity in the absence of an ACE inhibitor is known to be affected by genotypic variation, but genotype has very little effect on the measured serum ACE activity once the patient is taking an ACE inhibitor. In our previous work, serum ACE fell by only $3 \mathrm{EU} / 1$ when lisinopril dose was quadrupled from $5 \mathrm{mg}$ /day to $20 \mathrm{mg} /$ day. ${ }^{15}$

We found undetectable ACE and AII concentrations occurred in many patients being treated chronically with what would be considered "low" doses of ACE inhibitor. Between 5-20 mg enalapril equivalents, a low dose $(<10 \mathrm{mg} /$ day $)$ was not invariably associated with raised AII or aldosterone, nor was a high prescribed dose (>10 mg/day) a guarantee of "elimination" of AII or aldosterone in the presence of an ACE inhibitor drug. ${ }^{15}$ An average dose of an ACE inhibitor should virtually always eliminate ACE activity (always $<5 \mathrm{EU} / \mathrm{l}$ ) on chronic dosing (seven days). ${ }^{16}$ Anything less than this indicates poor compliance. There are currently remarkably few data on non-compliance with drug treatment in 
CHF patients, ${ }^{17}$ despite the fact that preliminary studies suggest that it may account for a substantial proportion of heart failure readmissions. ${ }^{18} 19$

In summary, this observational study revealed a wide diversity of AII and aldosterone suppression seen in routinely treated $\mathrm{CHF}$ patients. Many patients had normalised AII and aldosterone but few had undetectable concentrations and a substantial proportion had high normal or raised values. AII and aldosterone "reactivation" occurred in the presence of both high and low ACE concentrations, which suggests that different mechanisms contribute to hormonal "reactivation". In those with high ACE activity, non-compliance is likely to be the explanation. Since different mechanisms appear to produce AII "reactivation", it may be important to establish the precise mechanisms in each patient before deciding how to overcome hormone "reactivation" in each individual. Serum ACE concentrations may help to distinguish between the various possible causes of AII "reactivation" in individual patients and help the doctor to take the appropriate corrective action.

We are grateful to our clinical colleagues in the departments of medicine and cardiology at Ninewells Hospital, Dundee who allowed us to approach patients under their care to take part in allowed us to approach patients under their care to take part in
the study. We gratefully acknowledge the nursing support of the study. We gratefully acknowledge the nursing support of Jessamine Robson and excellent technical assistance of Mrs WJ Coutie and Mrs LM MacFarlane with hormone and electrolyte
analyses. This work was made possible by a grant from the analyses. This work was made possible by a grant fron
Scottish Office (acute health care research committee).

1 Clinical Quality Impact Network Investigators. Mortality risk and patterns of practice in 4606 acute care patients with congestive heart failure: the relative importance of age, sex and medical therapy. Arch Intern Med 1996;156:166973.

2 Packer M. New concepts in the pathophysiology of heart failure: beneficial and deleterious interaction of endogenous hemodynamic and neurohormonal mechanisms. $f$ Intern Med 1996;239:327-33.

3 Garg R, Yusuf S, for the Collaborative Group on ACE Inhibitor Trials. Overview of randomized trials of angio- tensin converting enzyme inhibitors on mortality and morbidity in patients with heart failure. $7 A M A 1995 ; 273: 1450$ 6.

4 Sigurdsson A, Held P, Andersson G, et al. Enalaprilat in acute myocardial infarction: tolerability and effects on the renin angiotensin system. Int F Cardiol 1991;33:115-24.

5 Juillerat L, Nussberger J, Menard J, et al. Determinants of angiotensin II generation during converting enzyme inhibition. Hypertension 1990;16:564-72.

6 Cleland JGF, Dutka DP, Ward S, et al. Effects of enalapril on clinical, non-invasive haemodynamic and neuroendocrine indices in elderly patients ( $>75$ years) with heart failure. Cardiology in the Elderly 1996;4:131-7.

7 Swedberg K, Enroth P, Kjekshus J, et al for the CONSENSUS Trial Study Group. Hormones regulating cardiovascular function in patients with severe congestive heart failure and their relation to mortality. Circulation 1990;82: 1730-6.

8 Belz GG, Kirsch W, Kleinbloesem CH. Angiotensin converting enzyme inhibitor: relationship between pharmacodynamics and pharmacokinetics. Clin Pharmacokinet 1988;15:295-318.

9 Fogarty Y, Fraser CG, Browning MC. Intra and inter individual variation of serum angiotensin converting enzyme: clinical implications. Ann Clin Biochem 1989;26:201-2.

10 Nussberger J, Waber G, Waeber B, et al. Plasma angiotensin 1-8 octapeptide measurement to assess acute angiotensin converting enzyme inhibition with captopril administered parenterally to normal subjects. F Cardiovasc Pharmacol 1988;11:716-21

11 Morton JJ, Webb DJ. Measurement of angiotensin II. Clinical Science 1985;68:483-4.

12 Norusis MJ. Statistical packages for the social sciences. In: SPSS for Microsoft Windows Advanced Statistics Release 6, Chicago; 1993.

13 Sigurdsson A, Swedberg K. The role of neurohormonal activation in chronic heart failure and post myocardial infarction. Am Heart f 1996;132:229-34.

14 Pitt B. Preliminary results of the randomized aldactone left ventricular failure evaluation study (RALES). Late breaking trials, American Heart Association annual scientific sessions. Dallas, Texas, November 1998.

15 Davidson NG, Coutie WJ, Webb DJ, et al. Hormonal and renal differences between low dose and high dose angiotensin converting enzyme inhibitor treatment in patients with chronic heart failure. Heart 1996;75:576-81.

16 MacFadyen RJ, Struther AD. The practical assessment of compliance with ACE inhibitor therapy: a novel approach. 7 Cardiovasc Pharmacol 1997;29:119-24.

17 Opasich C, Febo O, Riccardi G, et al. Concomitant factors of decompensation of heart failure. Am $\mathcal{F}$ Cardiol 1996;78: 354-7.

18 Rich MW, Vinson JM, Sperry JC, et al. Prevention of readmission in elderly patients with congestive heart failure: results of a prospective randomized pilot study. $\mathcal{F}$ Intern Med 1993;8:585-90.

19 Fox R. Compliance, adherence, concordance. Circulation 1998;97:127 